Between 1984 and 1989, cDNA sequence analyses of the whole TP53 coding region were successfully carried out in 370 patients operated on for primary breast cancer with axillary lymph node metastases. In total, 110 mutations were detected in 105 patients. Of these 110 mutations, 20 were located in exons 3-4 and $9-10$, and the remaining 90 were located in exons 5-8. Relapse-free survival, breastcancer-corrected survival and overall survival were analyzed in the subsets of patients receiving tamoxifen alone, CMF alone, or CMF plus tamoxifen. In node-positive breast cancer patients receiving CMF alone, the presence of TP53 mutations was found to be a statistically significant prognostic factor, correlating with worse relapse-free, breast-cancer-corrected and overall survival.

From these results, the authors conclude that women with mutations of TP53 and breast cancer should possibly be offered an alternative treatment to CMF therapy. Further investigation on the predictive therapeutic value of TP53 mutation is needed, however, and is currently being carried out in the randomized European Organisation for Research and Treatment of Cancer TP53 study.

\section{Marie Lofthouse}

Original article Andersson J et al. (2005) Worse survival for TP53 (p53)-mutated breast cancer patients receiving adjuvant CMF. Ann Oncol 16: 743-748

\section{Predicting outcome after preoperative therapy for rectal cancer}

Preoperative chemoradiation aims to minimize recurrence of cancer and enable more patients to proceed with tissue-sparing surgery. For patients with rectal cancer who achieve a partial response to preoperative chemoradiation, predicting long-term outcome can be difficult. Vecchio's group studied the ability of the TUMOR REGRESSION GRADE (TRG) system to predict outcome at 5 years for these patients.

This retrospective, single-center, Italian study included 144 patients, most of whom had clinical stage T3 or T4 and/or nodepositive rectal cancer. Patients underwent surgery 4-8 weeks after completion of radiotherapy and were followed up for at least 3 years. Proctoscopic biopsy specimens were obtained and examined both before treatment and 4-5 weeks after preoperative therapy ended. The degree of cytologic and stromal changes in the specimens led to assignment of TRG. Clinical response was evaluated by comparing pretreatment and post-treatment RECTAL REFERENCE INDEX SCORES.

This study suggests that, of all pretreatment and post-treatment factors, older age at diagnosis, low TRG and low pathologic nodal stage are statistically significant predictors of long-term survival. TRG was a more reliable indicator than other scoring methods such as digital rectal examination and tumor downstaging according to $\mathrm{WHO}$ criteria, with less interobserver variability. The authors acknowledged that the study's retrospective nature might have influenced the results, which should be validated in larger, prospective studies. Assessment of TRG might assist in identifying lower-risk patients for whom conservative surgery would be possible after preoperative radiotherapy.

\section{Rebecca Doherty}

Original article Vecchio FM et al. (2005) The relationship of pathologic tumor regression grade (TRG) and outcomes after preoperative therapy in rectal cancer. Int J Radiat Oncol Biol Phys 62: 752-760

\section{Response of malignant gliomas to erlotinib}

Long-term survival of patients with glioma has not improved, even with new treatment approaches. As the gene encoding the epidermal growth factor receptor (EGFR) is frequently amplified in gliomas, with concomitant increased levels of protein expression, inhibition of EGFR signaling could improve treatment. The EGFR inhibitor erlotinib shows clinical promise for treating malignant gliomas. In this phase I trial, Haas-Kogan et al. investigated the relationship between EGFR amplification, protein expression and response to erlotinib.

Patients were initially treated with $100 \mathrm{mg} /$ day erlotinib, increased by $50 \mathrm{mg} /$ day until doselimiting toxic effects occurred. They were then stratified into four groups according to the additional therapies received (temozolomide and/or enzyme-inducing antiepileptic drugs, or neither of these). Of 66 patients, 52 relapsed; brain tissue was acquired from 41 of these patients for evaluation of EGFR parameters.
GLOSSARY

TUMOR REGRESSION GRADE (TRG)

Histologic tumor regression score, graded from TRG1 (complete regression) to

TRG5 (no regression) on the basis of Mandard's criteria

RECTAL REFERENCE INDEX SCORE

Clinical response, scored as the number of quarters of the circumference of rectal wall affected by tumor (1-4) multiplied by length of tumor in $\mathrm{mm}$ 